\title{
The superdense plasma sheet in the magnetosphere during high-speed-stream-driven storms: Plasma transport timescales
}

\author{
Michael H. Denton ${ }^{\mathrm{a}, *}$, Joseph E. Borovsky ${ }^{\mathrm{b}}$ \\ a Department of Communication Systems, Lancaster University, Lancaster, UK \\ ${ }^{\mathrm{b}}$ Space Science and Applications, Los Alamos National Laboratory, Los Alamos, USA
}

\section{A R T I C L E I N F O}

\section{Article history:}

Accepted 18 April 2008

Available online 18 May 2008

Keywords:

Plasma sheet

Solar wind/magnetosphere interactions

Corotating streams

Magnetosphere: Inner

\begin{abstract}
A B S T R A C T
The superdense plasma sheet in the Earth's magnetosphere is studied via a superposition of multispacecraft data collected during 124 high-speed-stream-driven storms. The storm onsets tend to occur after the passage of the IMF sector reversal and before the passage of the stream interface, and the storms continue on for days during the passage of the highspeed stream. The superdense phase of the plasma sheet is found to be a common feature of high-speed-stream-driven storms, commencing before the onset of the storm and persisting for about 1 day into the storm. A separate phenomenon, the extra-hot phase of the plasma sheet, commences at storm onset and persists for several days during the storm. The superdense plasma sheet originates from the high-density compressed slow and fast solar wind of the corotating interaction region on the leading edge of the highspeed stream. Tracking the motion of this dense plasma into and through the magnetosphere, plasma transport times are estimated. Transport from the nightside of the dipole to the dayside requires about $10 \mathrm{~h}$. The occurrences of both the superdense plasma sheet and the extra-hot plasma sheet have broad implications for the physics of geomagnetic storms. (c) 2008 Elsevier Ltd. All rights reserved.
\end{abstract}

\section{Introduction}

The superdense plasma sheet was first noticed in the analysis of magnetotail and geosynchronous-orbit plasma data (Borovsky et al., 1997). It was found that at times the Earth's plasma-sheet density was anomalously high and that those "superdense" intervals were statistically distinct from the standard range of plasma-sheet densities. It was later learned that the superdense plasma sheet has its origin in anomalously high-density solar wind (Borovsky et al., 1998a), with the additional constraint that southward IMF is necessary for the dense solar-wind plasma to enter the magnetosphere (Thomsen et al., 1998a).

The "superdense" plasma sheet was defined in those early studies as a hot plasma-sheet plasma, to be

\footnotetext{
* Corresponding author. Tel.: +441524510544.

E-mail address: m.denton@lancaster.ac.uk (M.H. Denton).
}

contrasted with later studies of the "cool dense plasma sheet" which fills the outer magnetosphere during extended periods of northward IMF (e.g. Fujimoto et al., 2000, 2002, 2005; Phan et al., 2000; Nishino et al., 2002) and then is subsequently transported into the inner magnetosphere when geomagnetic activity commences (cf. Thomsen et al., 2003; Lavraud et al., 2005), particularly during high-speed-stream-driven storms preceded by calms (Borovsky and Steinberg, 2006; Lavraud et al., 2006).

In the superposed epoch studies of Denton and Borovsky (2008) the superdense plasma sheet is clearly seen during high-speed-stream-driven storms. Further, the superposed-epoch studies show that an anomalously hot ("extra-hot") plasma sheet is also a feature of highspeed-stream-driven storms (Denton et al., 2006; Denton and Borovsky, 2008).

In Fig. 1 the superdense plasma sheet and the extra-hot plasma sheet associated with high-speed-stream-driven storms can be seen. The plot is a superposition of 

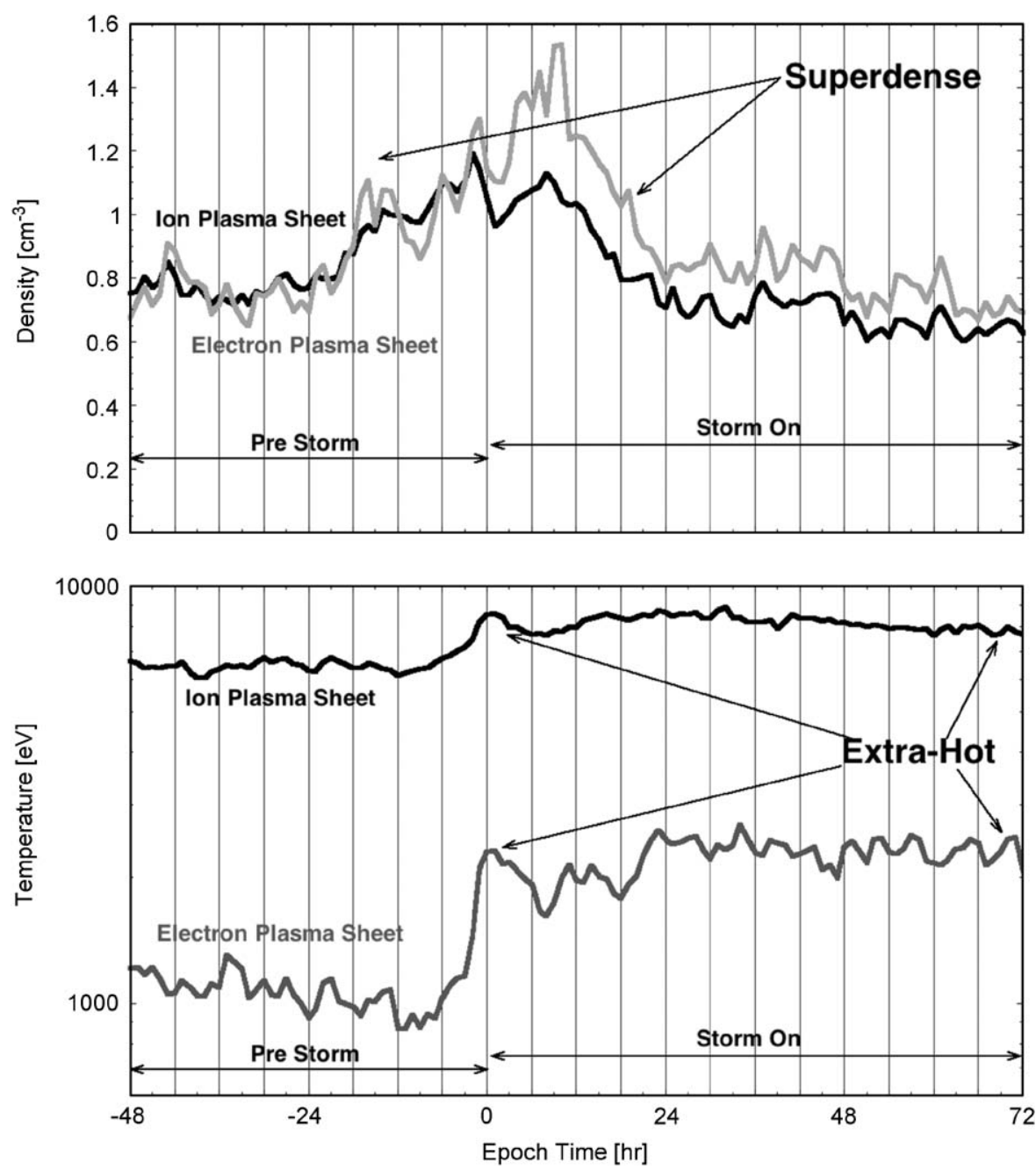

Fig. 1. In the top panel, for 124 high-speed-stream-driven storms the superposed-epoch average of the nightside (21-3 LT) hot-ion density (black) and hot-electron density (grey) is plotted as a function of the time from onset of storm. In the bottom panel, for 124 high-speed-stream-driven storms the superposed-epoch average of the hot ion temperature (black) and hot-electron temperature (grey) is plotted as a function of the time from onset of storm. The superdense phase of the plasma sheet is indicated in the top panel and the extra-hot phase of the plasma sheet is indicated in the bottom panel.

measurements taken by multiple satellites during 124 storms, with the zero epoch being the onset of storm levels of convection (see Section 2 for details of the data, the storms, and the superposition). In the top panel of Fig. 1 the hot-ion (0.1-45 keV: black line) and hotelectron (0.03-45 keV: grey line) densities measured at geosynchronous orbit in the nightside sector from 21 to 3 LT are plotted. The superposed average (mean) density clearly shows a superdense phase that starts prior to storm onset and lasts about 1 day. In the bottom panel of Fig. 1 the ion (black) and electron (grey) temperatures are plotted in the 21-3 LT nightside sector. The superposed average temperature shows an extra-hot plasma sheet that has a rapid onset at about storm onset and that persists for several days.

In this report the superdense plasma sheet during high-speed-stream-driven storms is studied in detail using superposed-epoch data analysis of solar-wind and magnetospheric measurements. It will be shown that the superdense plasma sheet is a common occurrence during the buildup and early phase of a high-speed-stream driven storm. It is associated with the passage of a corotating interaction region (CIR) at the leading edge of a high-speed stream, with the extra-dense magnetospheric plasma having its origin in the high density of the compressed solar-wind plasma of the CIR. It will be shown that the extra-hot plasma sheet commences when the storm commences and persists for days while the storm ensues. Timing the onset of the superdense plasma sheet at various locations in the magnetosphere will provide measurements of magnetospheric-convection timescales.

The superdense plasma sheet can be important for geomagnetic-storm physics. Its high density provides extra thermal energy with which to drive plasma waves in the magnetosphere that may be important for radiation-belt physics (Horne and Thorne, 1998; Summers and Thorne, 2003; Meredith et al., 2003). Its high pressure is 
associated with a diamagnetic distortion of the outer dipole (Borovsky et al., 1998b). And its high thermalenergy content might lead to stronger proton and diffuseelectron aurora, altering the properties of the stormtime ionosphere. The extra-hot plasma sheet is important for spacecraft charging during high-speed-stream-driven storms (Denton et al., 2006).

This manuscript is organized as follows. In Section 2 the event selection and data-analysis techniques are explained. In Section 3 the properties of the superdense plasma sheet are explored and the relation of the superdense plasma sheet to the properties of the solar wind is examined. Section 4 contains observations of the superdense plasma sheet used to measure mass-transport timescales within the magnetosphere. In Section 5 the onset of the extra-hot plasma sheet is explored in relation to the chronology of the solar wind and the geomagnetic storm. In Section 6 issues associated with the arrival of the superdense plasma sheet and the arrival of the extra-hot plasma sheet are discussed. The findings of this study are summarized in Section 7.

\section{Data sets and event selection}

As noted by Borovsky et al. (1998b): "in the near-Earth magnetotail, the gradient- and curvature-drift effects differ for plasma-sheet ions and plasma-sheet electrons ... as a result, the plasma sheet of the tail separates into an ion plasma sheet and an electron plasma sheet near the Earth, and the two plasma sheets have very different evolutions". At geosynchronous orbit, the ion and electron plasma sheets are not exactly co-located and they need not have the same number density. At geosynchronous orbit there are low-density populations of cool ions and electrons that can ensure charge neutrality.

The ion and electron plasma sheets in the dipolar magnetosphere are analysed using the multispacecraft MPA instruments (Bame et al., 1993), located on-board Los Alamos National Laboratory satellites in circular geosynchronous orbits $\left(6.6 R_{\mathrm{E}}\right)$ at the geographic equator. The MPA instruments measure three-dimensional ion and electron distribution functions every $86 \mathrm{~s}$ over the energy range $\sim 1 \mathrm{eV}$ to $45 \mathrm{keV}$. Each distribution function is acquired in $10 \mathrm{~s}$. Note that the ion temperatures measured by MPA are underestimates, owing to the fact that the ion plasma sheet contains a significant amount of ions with energies above the 45-keV limit of the detector (Borovsky et al., 1998c). The 1-min OMNI database (King and Papitashvili, 2005) is used to obtain the properties of the solar-wind plasma and the IMF at the magnetopause.

Recurring high-speed-streams make excellent candidates for superposed-epoch studies (e.g. McPherron and Weygand, 2006; Denton et al., 2006; Denton and Borovsky, 2008; Longden et al., 2008) because there is a repeating sequence of solar-wind types that pass the Earth (cf. Fig. 1 of Richardson et al., 1996): fast wind, rarefaction, slow wind, onset of CIR, compressed slow wind (westward flow), sector reversal within the compressed slow wind, stream interface, compressed fast wind (eastward flow), end of CIR, and again fast wind, rarefaction, etc. This repeating sequence is listed in the first column of Table 1.

The collection of high-speed-stream-driven storms used for the present study (1993-2006) is the set of 124 recurring storms used for the Denton and Borovsky (2008) superposed epoch study. The storms were found by using the McPherron list of solar-wind stream interfaces (Robert McPherron, private communication, 2007; McPherron and Weygand, 2006), with additions based on the same criteria, and then examining temporal plots of the Kp index to identify storms following the stream interfaces. The Kp index is used in preference to the Dst index since high-speed-stream-driven storms typically have poor Dst signatures (e.g. Kavanagh and Denton, 2007) Then, for each storm in the collection, the onset time of convection (storm onset) is determined as the first drop in Midnight Boundary Index (MBI) (Gussenhoven and Madden, 1990) of $>0.5^{\circ}$ latitude following the stream interface. MBI is an excellent indicator of magnetospheric convection, as is Kp (Thomsen, 2004), but MBI has higher time resolution than the 3-h Kp index. The storm onset times were determined to about 30-min accuracy.

\section{Properties of the superdense plasma sheet and relation to the solar wind}

In Fig. 2 superposed measurements from the magnetosphere and the solar wind are shown. The zero epoch of the plots is convection reaching storm levels. In the top panel of Fig. 2 the superposed average of the solar-wind speed is shown. At the initiation of the plot the solar wind speed is low (slow solar wind) and at the termination of the plot the solar-wind speed is high (fast solar wind).

Table 1

Typical chronology of a recurring-high-speed-stream cycle and the resulting geomagnetic activity and the behaviour of the Earth's plasma sheet

\begin{tabular}{|c|c|c|c|}
\hline The solar wind at earth & Stage of geomagnetic activity & Superdense status & Extra-hot status \\
\hline \multicolumn{4}{|l|}{ Rarefaction } \\
\hline Slow wind & Calm occurs $\sim 70 \%$ of time & Ordinary density & Ordinary temperature \\
\hline CIR starts & Calm ends & & \\
\hline \multicolumn{4}{|l|}{ Compressed slow wind (westward flow) } \\
\hline Sector reversal in compressed slow wind & Geomagnetic activity rises & Superdense starts & \\
\hline Compressed slow wind with southward IMF & Storm onset & & Extra-hot plasma sheet commences \\
\hline Stream interface (flow reversal) & & Superdense reaches dayside & \\
\hline Compressed fast wind (eastward flow) & Storm levels & & \\
\hline CIR ends & & Superdense ceases & \\
\hline Fast wind & Activity slowly declines & Ordinary density & Extra-hot persists \\
\hline
\end{tabular}


Between the slow wind and the fast wind is a CIR where the fast wind overtaking the slow wind forms a broad region of compressed plasma. The CIR is a region of enhanced pressure and density that spans the compressed

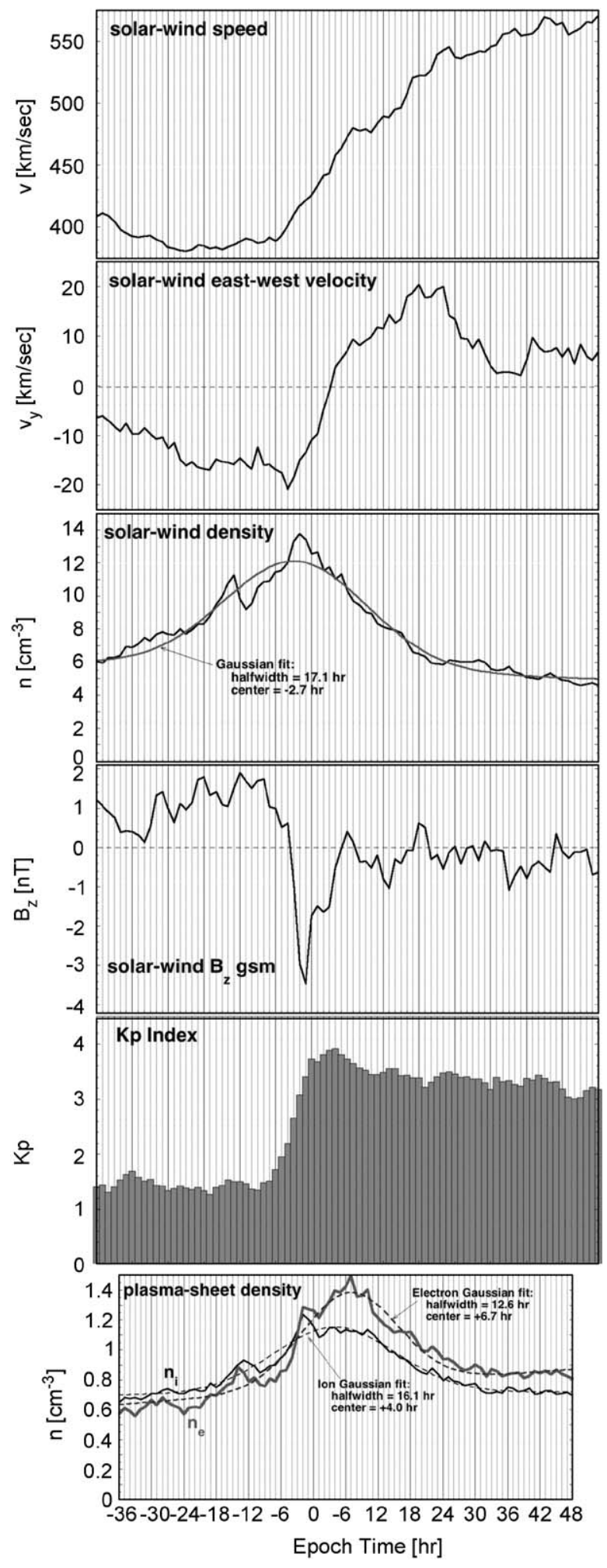

slow wind, the compressed fast wind, and the stream interface in between the two.

In the second panel of Fig. 2 the $y$-component (ecliptic) of the solar-wind flow velocity is plotted. Owing to the collision of fast and slow wind along an oblique front, there are east-west perturbations to the solar-wind radial outflow in the CIR. The compressed slow wind is characterized by a westward flow with density, temperature, and magnetic-field strength elevated above the preceding slow solar wind. The stream interface is marked by a reversal in the east-west flow direction: it is the interface between compressed slow wind and compressed fast wind. Following the stream interface the compressed fast wind is characterized by an eastward flow with a temperature and field strength further elevated from the compressed-slow-wind values but a density that is decreased from the compressed-slow-wind values. In the superposed-epoch-averaged data of the second panel of Fig. 2, the stream interface occurs at an epoch time $t=+3 \mathrm{~h}$.

In the third panel of Fig. 2 the superposed-epoch average of the solar-wind density is plotted as the black curve for the 124 storms. Note the enhanced density of the solar wind before and after the stream interface. This enhanced density is associated with the compression of the solar-wind plasma in the CIR; the earlier portion of the dense solar wind is compressed slow wind and the latter portion is compressed fast wind. A Gaussian fit to the enhanced density is plotted as the grey curve in the third panel (see Eq. (1), Section 4 for details of the fit). The full width of the Gaussian fit is $30 \mathrm{~h}$. This width is consistent with the width of the high-density solar-wind perturbations seen in the superposed-epoch study of McPherron and Weygand (2006), where the zero time of that superposition was chosen to be the solar-wind stream interface.

In the fourth panel of Fig. 2 the superposed average of the IMF component $B_{\mathrm{z}}$ (GSM) is plotted. Prior to the storm $B_{z}$ is predominantly northward (positive) and during the storm $B_{z}$ is predominantly southward (negative). A sector reversal typically lies several hours upstream of the stream interface (Wilcox and Ness, 1967). In the fourth panel, the superposed average would place the sector reversal at $t=-4 \mathrm{~h}$. Note that the Russell-McPherron effect prior to the sector reversal tends to produce a calm before the storm (Borovsky and Steinberg, 2006), if there is going to be a storm.

In the fifth panel of Fig. 2 the superposed average of the $\mathrm{Kp}$ index is plotted. Prior to the storm onset the

Fig. 2. For a superposition of measurements from 124 high-speedstream-driven storms, several quantities are plotted as a function of the time since storm onset. In the top panel the solar-wind speed is plotted in the second panel the $y$-component of the solar-wind velocity is plotted, in the third panel the density of the solar-wind plasma is plotted, in the fourth panel the $z$-component (GSM coordinates) of the solar-wind magnetic field is plotted, in the fifth panel the Kp index of geomagnetic activity is plotted, and in the bottom panel the density of the ion and electron plasma sheet on the nightside of the dipole (20-3 LT at geosynchronous orbit) is plotted. Gaussian fits to the solar-wind density and to the ion and electron plasma-sheet densities are shown in panels 3 and 6 . 
superposed average of the geomagnetic activity is modest and during the storm it is significantly higher.

In the bottom panel of Fig. 2 the density of the hot ions and the hot electrons averaged on the nightside of the magnetosphere from 20 to 3 LT is shown. The ion density is plotted as the solid black curve and the electron density is plotted as the solid grey curve. Prior to the storm the ion and electron densities are at normal levels; during the first day of the storm they are elevated to higher levels; and then later in the storm (the storms persist for several days; Denton and Borovsky, 2008) the plasma-sheet density returns to normal levels. Gaussian fits to the enhanced plasma sheet density are shown as the black (ion) and grey (electron) dashed curves in the bottom panel of Fig. 2. The full widths of the Gaussian fits are $32.6 \mathrm{~h}$ for the superdense ion plasma sheet and $26.2 \mathrm{~h}$ for the superdense electron plasma sheet. These values are indicators of the duration of a superdense plasma sheet in the nightside dipolar magnetosphere.

A comparison of the third and sixth panels of Fig. 2 demonstrates the relationship between the high-density solar wind and the superdense plasma sheet. The durations of the high-density solar wind $(\sim 30 \mathrm{~h})$ and super- dense ion $(\sim 33 \mathrm{~h})$ and electron $(\sim 26 \mathrm{~h})$ plasma sheets are similar, with the superdense plasma sheet appearing subsequent to the high-density solar wind. This is consistent with the findings of statistical comparisons of solar-wind and magnetospheric measurements (e.g. Terasawa et al., 1997; Borovsky et al., 1998a).

In Fig. 3 the superdense plasma sheet on the dayside of the Earth is compared with the superdense plasma sheet on the nightside. The top panel plots the hot-ion density in the 20-3 LT sector of geosynchronous orbit (black solid curve) and in the 9-15 LT sector (grey solid curve); Gaussian fits to these densities are shown as the dashed curves. In the bottom panel of Fig. 3 the hot-electron density in the 20-3 LT sector of geosynchronous orbit (black solid curve) and in the 9-15 LT sector (grey solid curve), with Gaussian fits to these densities shown as the dashed curves. As can be seen, the superdense phase is weaker and arrives later on the dayside as compared with the nightside. The superdense plasma sheet is particularly weak for electrons on the dayside (see also Section 3.2 of Borovsky et al., 1998b): plasma-sheet electrons are known to suffer strong losses in moving from the nightside to the dayside (Thomsen et al., 1998b).
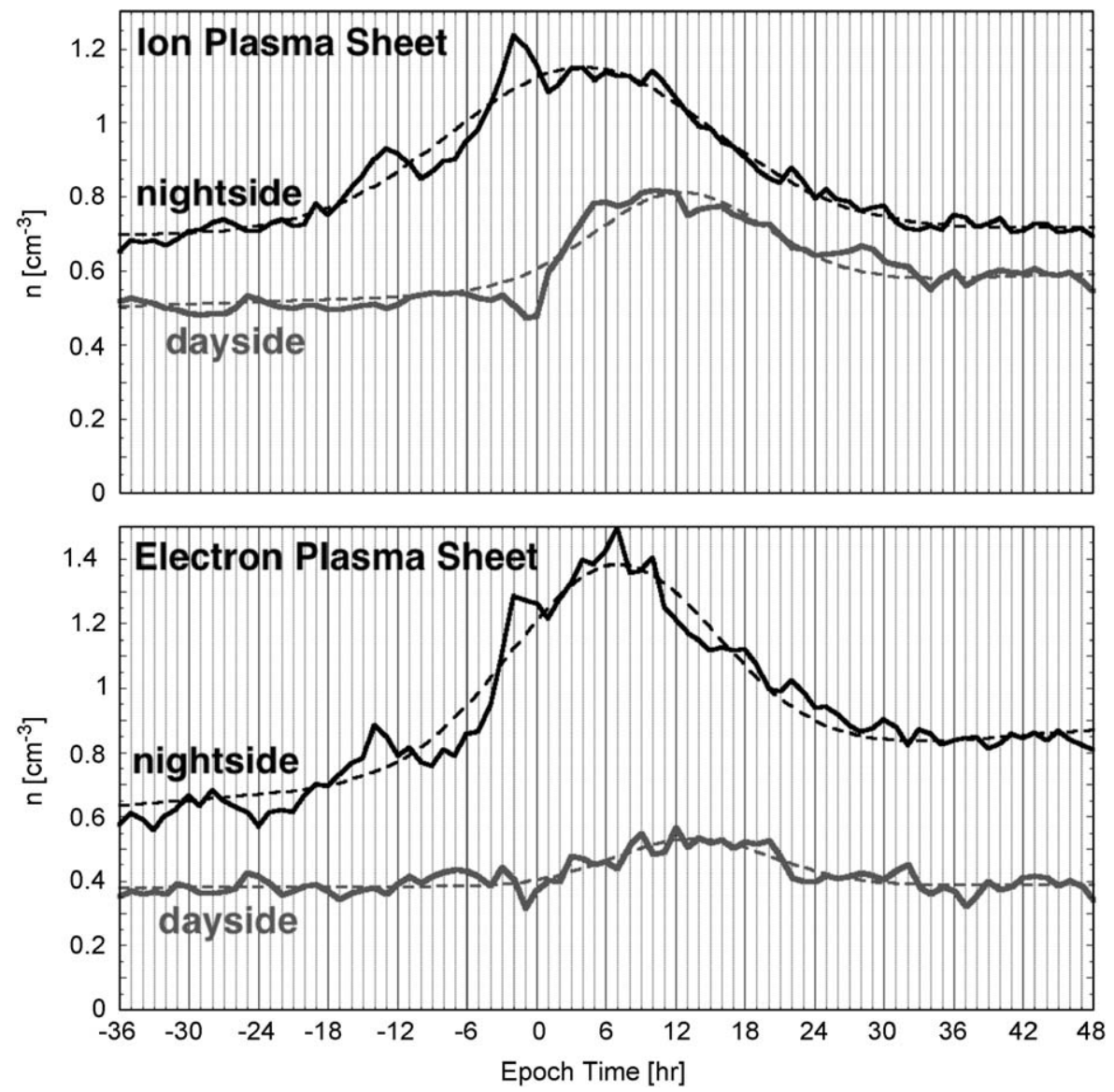

Fig. 3. In the top panel the superdense ion plasma sheet on the dayside (9-15 LT) is compared with the superdense ion plasma sheet on the nightside (20-3 LT). In the bottom panel the superdense electron plasma sheet on the dayside (9-15 LT) is compared with the superdense electron plasma sheet on the nightside (20-3 LT). All densities are the superposed-epoch averages of 124 high-speed-stream-driven storms. Gaussian fits are plotted as the dashed curves. 
In the first column of Table 1 the chronology of the passage of the CIR over the Earth is listed (cf. Fig. 1 of Richardson et al., 1996). In the second column of Table 1 the resulting reaction of the Earth in terms of geomagnetic activity is listed (cf. Table 1 of Borovsky and Steinberg, 2006). As noted in the table, geomagnetic calms occur prior to about $70 \%$ of high-speed-stream-driven storms. Table 1 notes that storm levels of activity are reached approximately when the stream interface passes the Earth: the second and fifth panels of Fig. 2 indicate that on average storm levels of activity are reached a few hours prior to the passage of the stream interface.

\section{Transport times in the magnetosphere}

To study mass-transport timescales within the magnetosphere, the movement of the superdense plasma sheet from the nightside to the dayside is timed in the superposed-epoch averages. Timing is analysed separately for the ion plasma sheet and the electron plasma sheet. Two methods are used. The first method looks at the timing at various locations of a fast rise in the density of the plasma sheet (similar to the single event analysed in Borovsky et al., 1998b mid-points of the fast rises at various local times are used to determine the timing). The
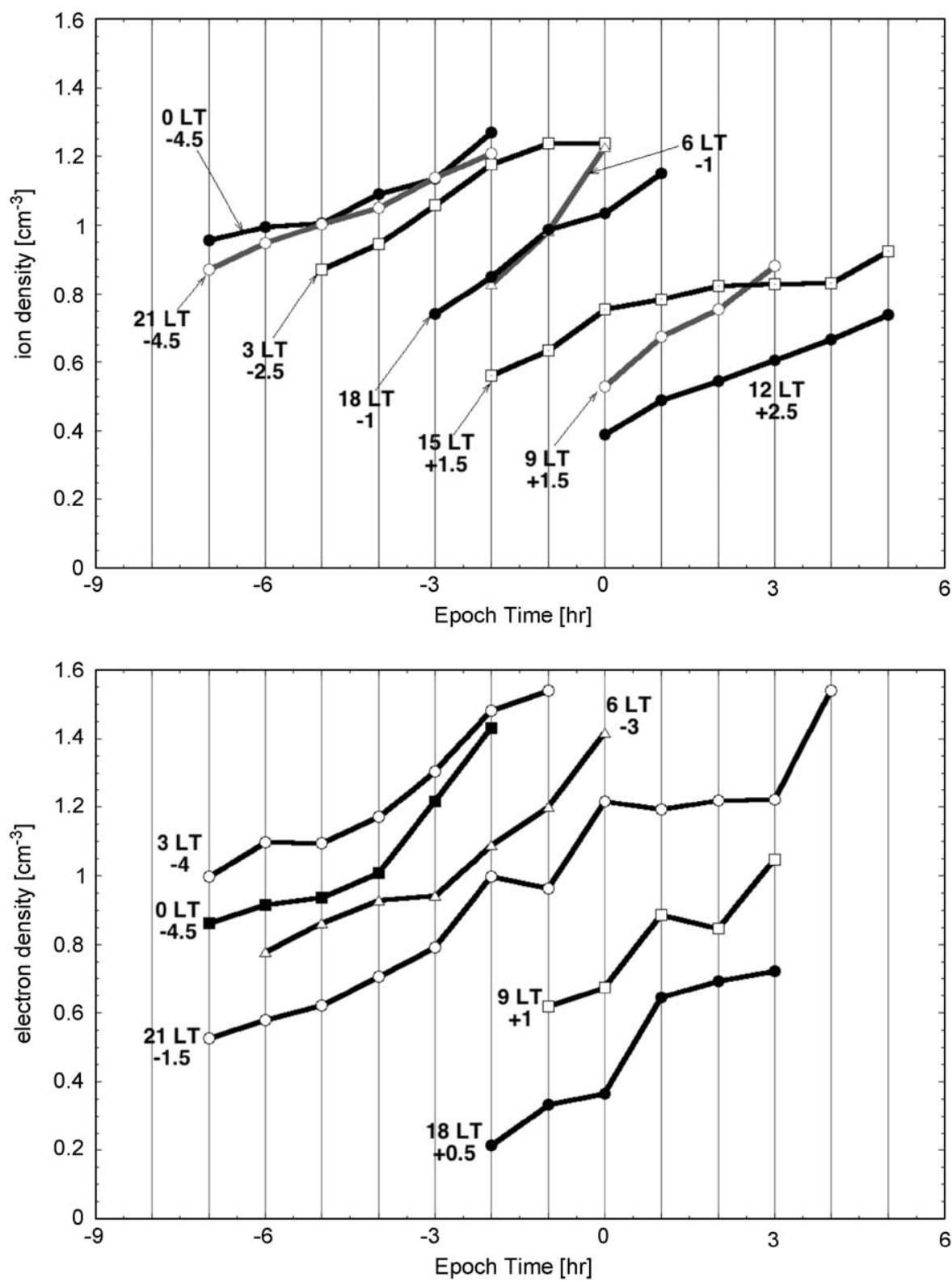

Fig. 4. From the superposed-epoch-averages of the ion density (top panel) and electron density (bottom panel) measured at geosynchronous orbit, the fast rises in the density are plotted at the various local times. These fast rises in density represent the transition to superdense plasma sheet from ordinary plasma sheet. The mean epoch time of the fast rise is also labelled. Note: the choice of symbols and line colour to help distinguish between each local time. 
second method looks at the time shifts between Gaussian fits to the plasma-sheet density at various local times.

In the two panels of Fig. 4, the fast rises in the ion density (top panel) and electron density (bottom panel) at various local times are plotted. These fast rises in the number density represent the onset of the superdense plasma sheet at the various local-time locations around the Earth. Each curve is the average of a local-time sector that is $3 \mathrm{~h}$ wide. The various curves are labelled according to (a) the central local time of the 3-h-wide sector and (b) the mean epoch time of the fast rise. Note that the vertical and horizontal scales are the same for the two plots in Fig. 4. Two clear local-time trends can be seen in both plots in Fig. 4: (1) the superdense plasma sheet is less dense on the dayside than it is on the nightside and (2) the superdense plasma sheet appears first on the nightside and then later on the dayside. Measuring the fast rise in the solar-wind density at Earth (not shown) yields a time of $-6.5 \mathrm{~h}$. In the superposed averages, there is no rise in the hot-electron density seen in the 12 and 15 LT sectors. Early in high-speed-stream-driven storms these two localtime sectors tend to be filled with broad plasmaspheric drainage plumes (Borovsky and Denton, 2008; Denton and Borovsky, 2008) and it is known that the electron plasma sheet and the plasmasphere rarely interpenetrate (cf. Thomsen et al., 1998b).
In panels $\mathrm{a}$ and $\mathrm{b}$ of Fig. 5, the transport timescales for the movement of the superdense plasma sheet around the Earth is summarized. Each temporal value marked in the sketch is the delay in time at which the superdense plasma sheet arrives at that location relative to the time it arrived at local midnight. The temporal values marked in panel a of Fig. 5 are obtained from the top panel of Fig. 4 and the values marked in panel b of Fig. 5 are obtained from the bottom panel of Fig. 4 .

In panel $\mathrm{a}$ and $\mathrm{b}$ of Fig. 6 the delay in rise times from the fast rise in the solar-wind density at Earth are shown. (The rise in the solar-wind density occurred at epoch time $t=-6.5 \mathrm{~h}$.) Panel a pertains to the arrival of the superdense ion plasma sheet relative to the rise in solar-wind density and panel $b$ pertains to the arrival of the superdense electron plasma sheet relative to the rise in the solar-wind density.

The second method used to look at the timing of plasma transport is to compare Gaussian fits to the enhanced density of the plasma sheet at various local times and to the solar-wind density. Gaussian fits (cf. panels $c$ and $f$ of Fig. 2) of the density $n$ as a function of epoch time $t$ of the form

$n=C_{1}+C_{2} \exp \left[-\left(\frac{t-C_{3}}{C_{4}}\right)^{2}\right]+C_{5} t$ a

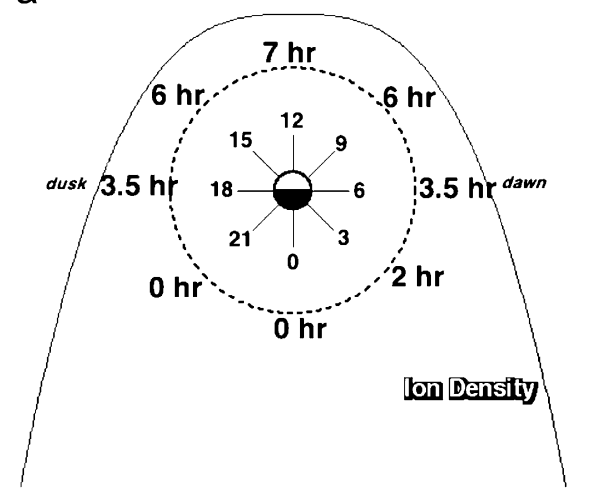

C

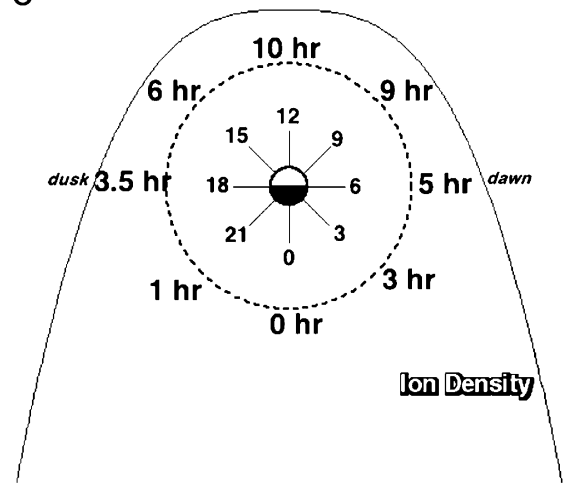

b

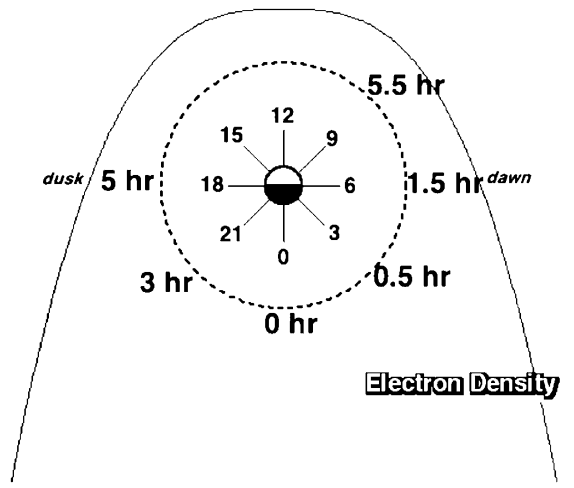

d

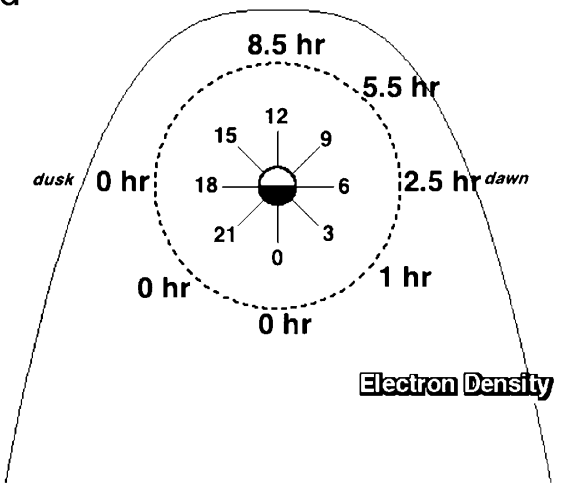

Fig. 5. The transport times for ions and electrons from local midnight to other local times around geosynchronous orbit are summarized for the two methods used: the fast-rise method ( $a$ and $b$ ) and the Gaussian-fit method ( $c$ and d). Both methods use the superposed-epoch-averaged density measurements for the 124 high-speed-stream-driven storms. 
a

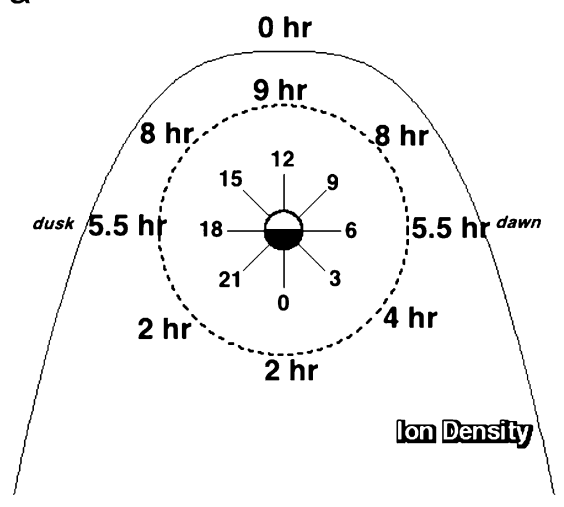

C

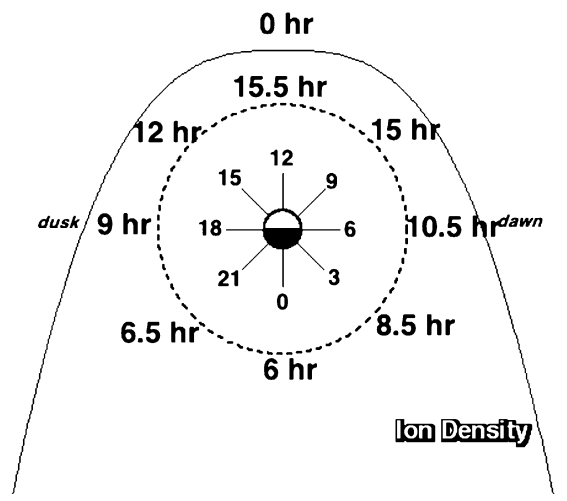

b

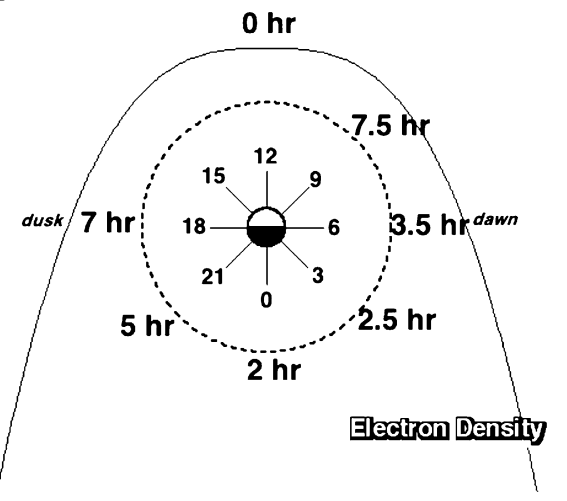

d

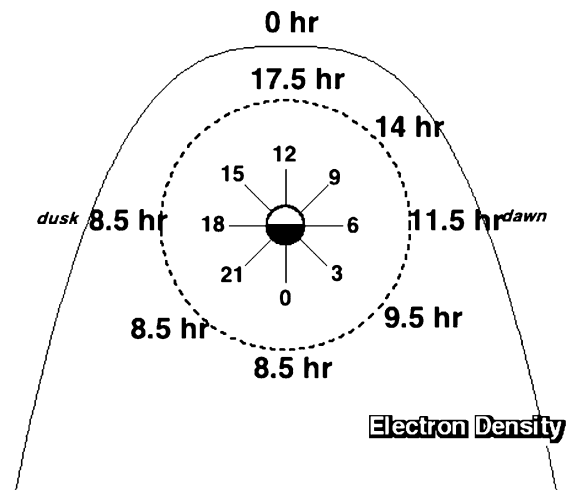

Fig. 6. The transport times for ions and electrons from the solar wind (at Earth) to the various local times around geosynchronous orbit are summarized for the two methods used: the fast-rise method (a and b) and the Gaussian-fit method (c and d). Both methods use the superposed-epoch-averaged density measurements for the 124 high-speed-stream-driven storms.

are made, where $C_{1}, C_{2}, C_{3}, C_{4}$, and $C_{5}$ are constants that are determined by a least-squares method. The constants of interest are $C_{2}$ (the amplitude of the density perturbation), $C_{3}$ (the epoch time upon which the Gaussian fit is centred), and $C_{4}$ (the halfwidth of the Gaussian). $C_{1}$ is the average density of the plasma sheet before and after the superdense plasma sheet and the term $C_{5} t$ in expression (1) allows for a change in plasma sheet density before and after the superdense phase. The fits are performed over the time range from -36 to $+48 \mathrm{~h}$. The relevant parameters for Gaussian fits to the solar wind density, to the hot-ion density at various local times at geosynchronous orbit, and to the hot-electron density at various local times at geosynchronous orbit (where available) are collected into Table 2 .

In panels $c$ and $d$ of Figs. 5 and 6 the centre times of the Gaussian fits are used to examine the transport times within (Fig. 5) and into and through (Fig. 6) the magnetosphere. In Fig. 5 the times in the sketches are the centre times of the various Gaussian fits relative to the centre time at local midnight and in Fig. 6 the times in the sketches are the centre times of the various Gaussian fits relative to the centre time of the Gaussian fit to the solarwind density.

Comparing panels $\mathrm{a}$ and $\mathrm{b}$ of Fig. 5 with panels $\mathrm{c}$ and $\mathrm{d}$ of Fig. 5, one can see that the two methods (tracking the fast rise in density and comparing the centres of the Gaussian fits) yield similar values for the plasma-transport times around the Earth. The hot ions and hot electrons appear first at midnight and then take $10 \mathrm{~h}$ or less to reach the dayside. Electron transport to the dayside is chiefly along the dawnside. Ion transport to the dayside appears to be via both the dawn and dusk sectors. This dawn and dusk ion transport is consistent with the findings of Denton and Borovsky (2008) that ion flows during high-speed-stream-driven storms are sunward at about $6 \mathrm{~km} / \mathrm{s}$ in both the dawn and dusk sectors of geosynchronous orbit. At $6 \mathrm{~km} / \mathrm{s}$, transport from midnight to noon along the circle of geosynchronous orbit would require $\left(1.31 \times 10^{5} \mathrm{~km}\right) /(6 \mathrm{~km} / \mathrm{s}) \sim 6 \mathrm{~h}$. This 6 -h estimate is consistent with the values found in the present study. Note that the transport-time values arrived at in the present study are considerably larger than values estimated using traces of (Volland-Stern) ionospheric-convection patterns (Borovsky et al., 1998c), which yielded $4.2 \mathrm{~h}$ or less from transport from the magnetotail to the dayside neutral line, meaning less than $4.2 \mathrm{~h}$ for transport from midnight geosynchronous to noon geosynchronous.

Comparing panels $\mathrm{a}$ and $\mathrm{b}$ of Fig. 6 with panels $\mathrm{c}$ and $\mathrm{d}$ of Fig. 6, one finds that the fast-rise method obtains a quicker estimate of the timescale for solar wind plasma to enter the magnetosphere and reach midnight (about $2 \mathrm{~h}$ ) 
Table 2

Parameters for Gaussian fits to the solar-wind density and the hot-ion density and hot-electron density at various local-time locations around geosynchronous orbit (where available)

\begin{tabular}{|c|c|c|c|c|c|}
\hline Function fit & $\begin{array}{l}\text { Gaussian amplitude } \\
\left(\mathrm{cm}^{-3}\right)\end{array}$ & $\begin{array}{l}\text { Gaussian fullwidth } \\
\text { (h) }\end{array}$ & $\begin{array}{l}\text { Center of Gaussian } \\
\text { (h) }\end{array}$ & $\begin{array}{l}\text { Time from solar } \\
\text { wind }(\mathrm{h})\end{array}$ & $\begin{array}{l}\text { Time from local } \\
\text { midnight }(\mathrm{h})\end{array}$ \\
\hline \multicolumn{6}{|l|}{ Solar wind } \\
\hline$n_{\mathrm{sw}}$ & 6.5 & 34.2 & -2.7 & 0 & \\
\hline \multicolumn{6}{|c|}{ Ion plasma sheet } \\
\hline$n_{\mathrm{i}} 0 \mathrm{LT}$ & 0.43 & 35.2 & 3.1 & 5.9 & 0 \\
\hline$n_{\mathrm{i}} 3 \mathrm{LT}$ & 0.49 & 29.2 & 5.8 & 8.6 & 2.7 \\
\hline$n_{\mathrm{i}} 6 \mathrm{LT}$ & 0.55 & 24.7 & 8.0 & 10.7 & 4.9 \\
\hline$n_{\mathrm{i}} 9 \mathrm{LT}$ & 0.35 & 22.6 & 12.1 & 14.8 & 9.0 \\
\hline$n_{\mathrm{i}} 12 \mathrm{LT}$ & 0.23 & 19.3 & 13.0 & 15.8 & 9.9 \\
\hline$n_{\mathrm{i}} 15 \mathrm{LT}$ & 0.29 & 20.0 & 9.1 & 11.8 & 5.9 \\
\hline$n_{\mathrm{i}} 18 \mathrm{LT}$ & 0.46 & 21.6 & 6.5 & 9.2 & 3.4 \\
\hline$n_{\mathrm{i}} 21 \mathrm{LT}$ & 0.45 & 29.9 & 3.7 & 6.5 & 0.6 \\
\hline \multicolumn{6}{|c|}{ Electron plasma sheet } \\
\hline$n_{\mathrm{e}} 0 \mathrm{LT}$ & 0.57 & 30.8 & 6.0 & 8.7 & 0 \\
\hline$n_{\mathrm{e}} 3 \mathrm{LT}$ & 0.69 & 30.1 & 6.9 & 9.6 & 0.9 \\
\hline$n_{\mathrm{e}} 6 \mathrm{LT}$ & 0.71 & 25.6 & 8.6 & 11.3 & 2.6 \\
\hline$n_{\mathrm{e}} 9 \mathrm{LT}$ & 0.44 & 24.8 & 11.3 & 14.0 & 5.3 \\
\hline$n_{\mathrm{e}} 12 \mathrm{LT}$ & 0.12 & 15.7 & 14.6 & 17.3 & 8.6 \\
\hline \multicolumn{6}{|l|}{$n_{\mathrm{e}} 15 \mathrm{LT}$} \\
\hline$n_{\mathrm{e}} 18 \mathrm{LT}$ & 0.34 & 11.8 & 5.7 & 8.5 & -0.2 \\
\hline$n_{\mathrm{e}} 21 \mathrm{LT}$ & 0.73 & 16.7 & 5.9 & 8.6 & -0.1 \\
\hline
\end{tabular}

The fits are over epoch time -48 to $+48 \mathrm{~h}$.

than does the Gaussian-fit method (about 6-8.5 h). The authors feel that the fast-rise method is a more direct method for measuring plasma-transport times.

In Fig. 7 a sketch summarizing transport times from Borovsky et al. (1998b) is presented. Those transport times were obtained by cross correlating solar-wind density with magnetospheric hot-ion density, varying the time lag between the solar-wind measurements and the magnetospheric measurements, and taking the time lag that yields the largest linear correlation coefficient to be the transport time. The Borovsky et al. (1998a) data sets had a mixture of non-storm and storm times and a mixture of ordinary plasma sheet and superdense plasma sheet, with the predominance of data for non-storm and ordinary plasma sheet. As can be seen by comparing Fig. 7 with Fig. 6, the transport-time values of Borovsky et al. (1998a), particular those for solar wind to midnight geosynchronous and from solar wind to noon geosynchronous, are in the middle of the spread in values obtained in the present study.

The timing of the onset and end of the superdense plasma sheet relative to the chronologies of the solar wind and the storm is summarized in the two panels of Fig. 8. The top panel pertains to the results of the fast-rise method and the bottom panel pertains to the results of the Gaussian-fit method. The values plotted in the top panel come from Fig. 4 and the values plotted in the bottom panel come from Table 2. In the top panel the solid green curve plots the onset time of the superdense ion plasma sheet as a function of the local time at geosynchronous orbit. The solid blue curve is the arrival time of the superdense electron plasma sheet as a function of local time. Various times in the storm and the solar wind

\section{Ion Ttransport Theses from Gross Correlations}

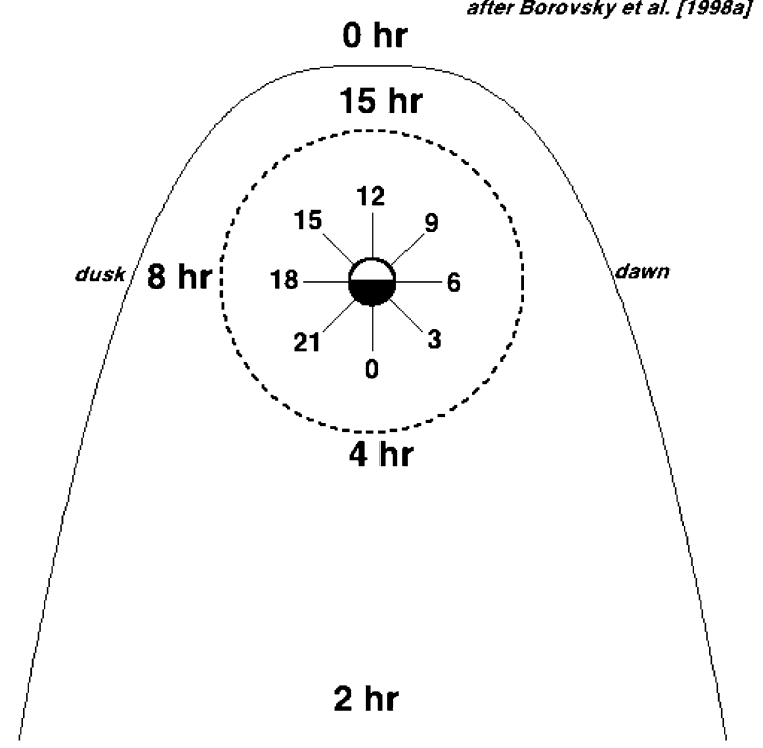

Fig. 7. The transport times into and through the magnetosphere obtained by Borovsky et al. (1998a) using cross-correlation methods on density measurements is summarized. The " $2 \mathrm{~h}$ " label represents the centre of the magnetotail plasma sheet at $X=-20 R_{\mathrm{E}}$.

are marked with the horizontal dashed lines. In particular, the time of the fast rise in the solar-wind density is marked with a red dashed line. In the top panel the appearance of the superdense plasma sheet first at midnight and then at later times around the dipole is 

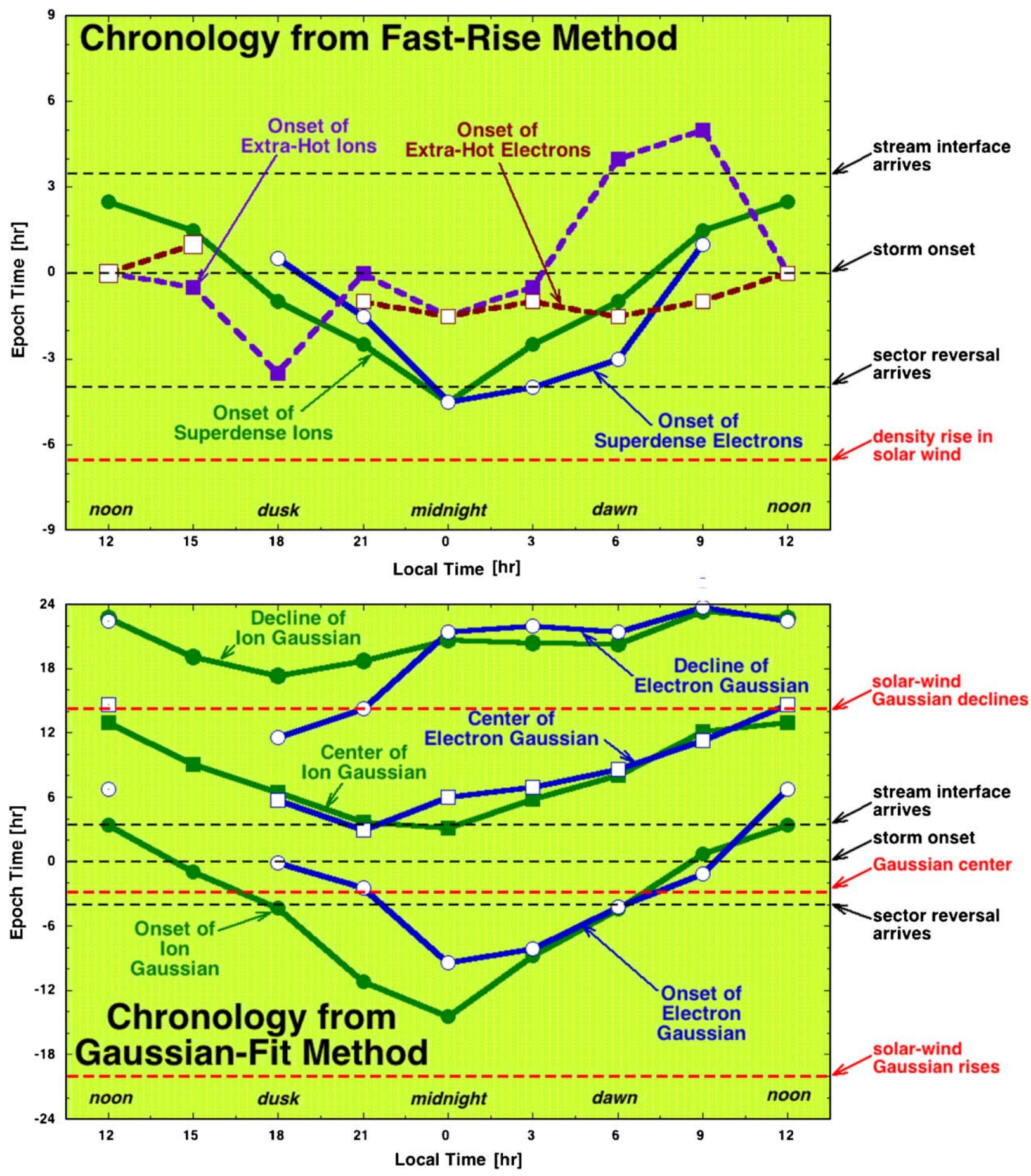

Fig. 8. The timing of the superdense plasma sheet and the extra-hot plasma sheet are summarized and compared with storm and solar-wind timing. In the top panel the arrival times of the superdense ion (green) and superdense electron (red) plasma sheets and the extra-hot ion (purple) and extra-hot electron (brown) plasma sheets are plotted as functions of local time. In the bottom panel the initiation, centre, and decline of the superdense ion plasma sheet (green curves) and the superdense electron plasma sheet (blue curves) are plotted as functions of local time. The values plotted in the top panel come from Figs. 4 and 9; the values plotted in the bottom panel come from Table 2. In both panels various solar wind and storm characteristic times are indicated by the vertical dashed lines.

clearly seen. This first appearance in the magnetosphere is after the appearance in the solar wind (red dashed line). The appearance of the superdense plasma sheet at midnight is about the time of passage of the IMF sector reversal in the solar wind; perhaps not coincidentally, this passage signals the onset of geomagnetic activity.

In the plot in the bottom panel of Fig. 8 the onset and disappearance of the superdense plasma sheet is 
examined using the Gaussian fits to the enhanced density (see Table 2). This plot spans more epoch time than the plot in the top panel. The three solid green curves mark the arrival, centre, and departure of the ion plasma sheet as determined by the centre time and halfwidths of the Gaussian fits to the ion density at the various local times. Likewise, the three solid blue curves mark the arrival, centre, and departure of the electron plasma sheet as determined by the centre time and halfwidths of the Gaussian fits to the electron density at the various local times. The three horizontal red dashed lines in the bottom panel are the arrival, centre, and departure of the high-density solar wind as determined from the centre time and halfwidths of the
Gaussian fit to the solar-wind density. The bottom panel of Fig. 8 also shows that the superdense plasma sheet appears at local midnight after it appears in the solar wind and that the superdense plasma sheet spreads to other local times after its appearance at midnight. And as can be seen, the decline of the superdense plasma sheet also occurs after the decline in the solar-wind density.

\section{Arrival of the extra-hot plasma sheet}

As demonstrated in the top panel of Fig. 1, the ion and electron plasma sheets have a superdense phase that lasts for about 1 day early in a high-speed-stream-driven
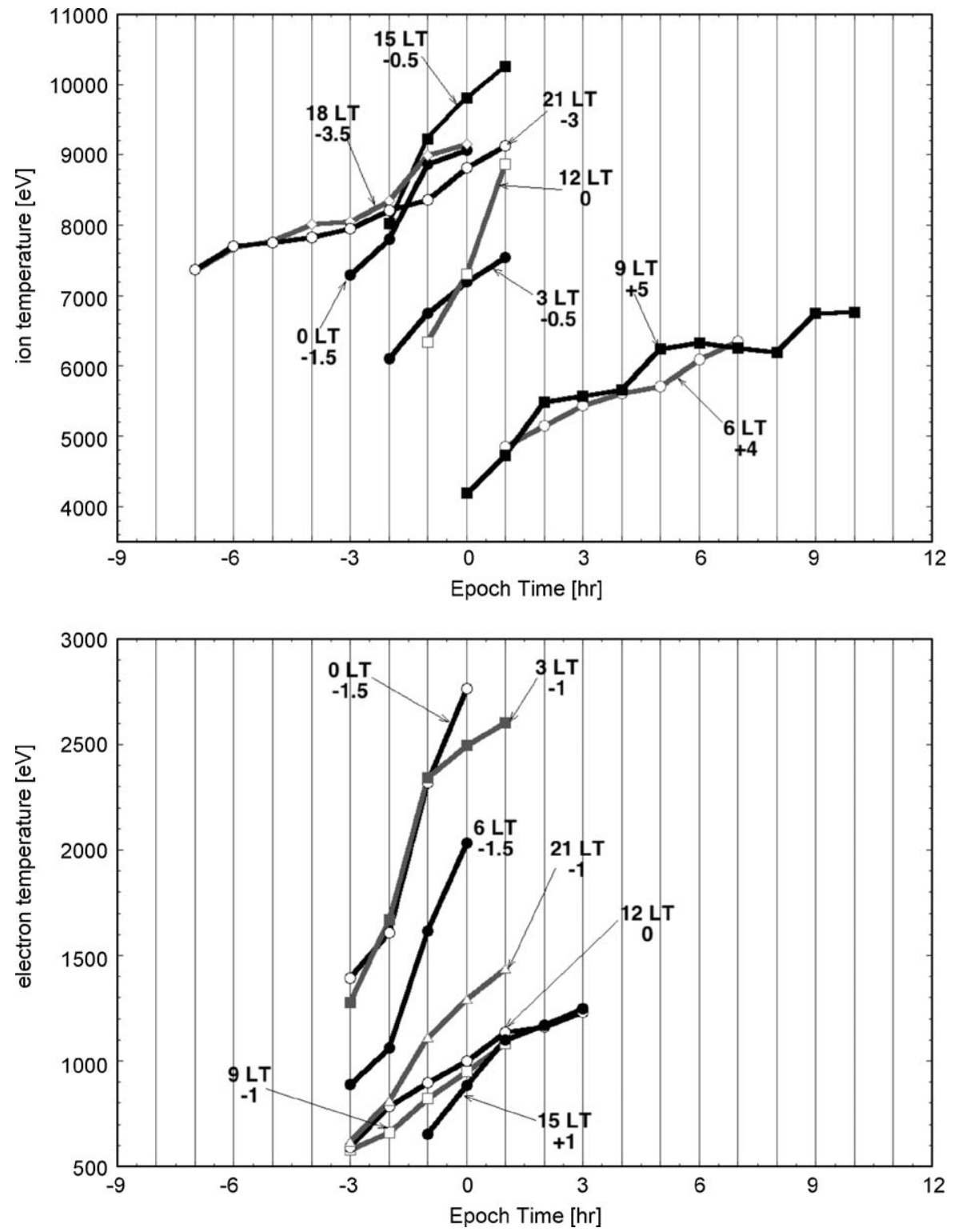

Fig. 9. From the superposed-epoch-averages of the ion temperature (top panel) and electron temperature (bottom panel) measured at geosynchronous orbit, the fast rises in the temperatures are plotted at the various local times. These fast rises in temperature represent the transition to extra-hot plasma sheet from plasma sheet with normal temperatures. The mean epoch time of the fast rise is also labelled. Note: the choice of symbols and line colour to help distinguish between each local time. 
storm. And as demonstrated in the bottom panel of Fig. 1, the ion and electron plasma sheets have an extrahot phase that lasts for several days during a high-speedstream-driven storm.

The extra-hot electron plasma sheet is the cause of the high levels of spacecraft charging measured during highspeed-stream-driven storms (Borovsky et al., 1998b; Denton et al., 2006). Note that the extra-hot ion plasma sheet provides a poor fuel for the ring current; gradientand curvature-drift effects prevent the extra-hot ions from convecting deep into the dipole where their pressure can be amplified (e.g. Ebihara and Ejiri, 2000; Liemohn et al., 2001; Kozyra and Liemohn, 2003). The extra-hot nature of the plasma sheet probably contributes to the fact that high-speed-stream-driven storms tend to have poor Dst signatures (Borovsky and Denton, 2006; see also McPherron and Weygand, 2006; Tsurutani et al., 2006).

In Fig. 9 the rapid transition to the extra-hot phase is examined in the various 3-h-wide local-time sectors. The top panel of Fig. 9 plots the transitions for the ion plasma sheet and the bottom panel plots the transitions for the electron plasma sheet. The various curves are labelled according to (a) the central local time of the sector and (b) the mean epoch time of the ramp. The horizontal axes are the same for the two plots, but the vertical axes differ: the ions are much hotter (cf. Fig. 1). Both panels show the trend that the extra-hot plasma sheet first appears on the nightside and then later appears on the dayside. As can be seen by comparing the two panels of Fig. 9, the transition to the extra-hot plasma sheet is globally more rapid for the electrons, with only about a 2.5 -h spread in arrival time for all local times. The transition to the extra-hot phase for the ions takes about $7.5 \mathrm{~h}$ from nightside to dayside.

The top panel of Fig. 8 demonstrates that the arrival of the extra-hot phase is not simultaneous with the arrival of the superdense phase. Here, the first appearance of the extra-hot ion plasma sheet (red) and extra-hot electron plasma sheet (purple) are plotted along with the first appearance of the superdense ion plasma sheet (green) and the superdense electron plasma sheet (brown), all as functions of local time. At the various local times, the arrival times of superdense and the arrival times of extrahot are not simultaneous. The values for these four curves in Fig. 8 come from Figs. 4 and 9. As can be seen, on the nightside of the Earth the superdense phase precedes the extra-hot phase. Note also that the arrival times of ion and electron superdense are not simultaneous (see also Borovsky et al., 1997) and that the arrival times of ion and electron extra-hot are not simultaneous.

\section{Discussion}

In this section, the timing of the onset of the superdense plasma sheet (Section 6.1) and the timing of the onset of the extra-hot plasma sheet (Section 6.2) are discussed.

\subsection{On the entry of superdense plasma into the magnetosphere}

In the analysis of the superposed-epoch averaged solarwind and magnetospheric data using rise-time and Gaus- sian-fit methods, the transport-time values from the solar wind to geosynchronous midnight vary considerably, from 2 to $8.5 \mathrm{~h}$ (see Fig. 6). The Borovsky et al. (1998a) study, using cross-correlation methods, obtained $4 \mathrm{~h}$ for the solar wind to geosynchronous midnight transport (see Fig. 7). These transport times represent the time lag between a change in the solar-wind density and a change in the magnetospheric (midnight) density. If another factor acts to control the entry of solar-wind plasma into the magnetosphere (such as whether or not the IMF is southward) then a simple density of the solar wind comparison with the density in the magnetosphere is insufficient to measure the transport time. A more-sophisticated analysis in future looking at solar-wind density, magnetospheric density, and the IMF should be performed to investigate the regulation and the transport times.

\subsection{On the rapid transition to the extra-hot plasma sheet}

One might guess that the rapid transition to the extrahot plasma sheet might be associated with a slow-solarwind to fast-solar-wind transition at perhaps the stream interface. This simple association is not the case. As can be seen in Fig. 8, the rapid transition to extra-hot plasma sheet occurs in the magnetosphere prior to the arrival of the stream interface, at least as seen in superposed-epoch averages. The arrival of the extra-hot plasma sheet is more associated with the onset of storm levels of magnetospheric convection. At this time (epoch time $=0 \mathrm{~h}$ ) the solar-wind velocity is still quite low (see top panel of Fig. 2). The extra-hot phase persists as the solar-wind velocity increases and persists for days as the high-speed stream continues to drive activity. To ensure that the arrival of the extra-hot plasma sheet is indeed prior to the passage of the stream interface a future study of the extrahot plasma sheet in high-speed-stream-driven storms should be performed with the zero of superposed epoch chosen to the solar-wind stream interface.

\section{Summary}

In this report the superdense plasma sheet and the extra-hot plasma sheet were studied during high-speedstream-driven storms using a superposed-epoch analysis of solar wind and magnetospheric measurements. The basic findings of this study are the following:

(1) The onset of the superdense plasma sheet in the magnetosphere is prior to storm onset. The superdense plasma sheet first appears about the time of passage of the sector reversal.

(2) The superdense plasma sheet arrives on the nightside first, later at dawn and dusk, and finally it arrives on the dayside. The transport of the ion plasma sheet from midnight to the dayside appears to be via both the dawn and dusk sectors; the transport of the electron plasma sheet from midnight to the dayside appears to be via the dawn sector.

(3) The superdense ion plasma sheet and the superdense electron plasma sheet do not commence simultaneously. 
At some local times the superdense ions arrive first, at other local times the superdense electrons arrive first.

(4) The superdense plasma sheet lasts for about a day and a half. The superdense plasma sheet is a feature of the early phase of a high-speed-stream-driven storm.

(5) The origin of the superdense plasma sheet appears to be the compressed slow and fast wind of the CIR. The onset and termination of the superdense plasma sheet in the magnetosphere both lag the onset and termination of the high-density plasma of the solar wind.

(6) The extra-hot plasma sheet commences approximately at storm onset. This commencement is prior to the passage of the stream interface, which is the nominal interface between slow and fast wind. Hence the extra-hot plasma sheet arrives prior to what is commonly defined as fast wind.

(7) The extra-hot ion plasma sheet and the extra-hot electron plasma sheet do not commence simultaneously. At some local times the extra-hot ions arrive first, at other local times the extra-hot electrons arrive first.

(8) The extra-hot plasma sheet lasts for several days during a high-speed-stream-driven storm. The extra-hot phase persists, on average, for at least 4 days after the onset of each storm.

(9) Measured plasma-transport timescales in the magnetosphere are $7-10 \mathrm{~h}$ for plasma to travel from the nightside to the dayside. These transport times agree approximately with plasma flow velocity measurements at geosynchronous orbit. These transport times are considerably longer than transport times inferred from an ionospheric convection model.

(10) Values obtained for the timescale for solar-wind plasma to enter the magnetosphere and reach geosynchronous orbit vary from 2 to $8.5 \mathrm{~h}$. Different methods on the hot ions and hot electrons yield different values. The timescale is ambiguous in this study.

A summary of the chronology of the storm, the superdense plasma sheet, and the extra-hot plasma sheet is the following (see also Table 1). Storm onset occurs in the compressed slow wind a few hours after the sector reversal passes the Earth and prior to the passage of the stream interface. The storm then persists for days. The superdense plasma sheet appears in the magnetosphere prior to storm onset and persists for about 1 day into the storm. The extra-hot plasma sheet commences with storm onset and persists for several days during the storm.

The superdense plasma sheet may be important for driving magnetospheric plasma waves, for distorting the outer dipole, and for producing strong proton and diffuseelectron aurora. The extra-hot plasma sheet is important for high levels of spacecraft charging and may be important for limiting the Dst perturbation of highspeed-stream-driven storms.

\section{Acknowledgements}

We wish to thank Bob McPherron for providing the initial list of stream interfaces and Michelle Thomsen for helpful discussions. JEB wishes to thank the Department of Communication Systems at Lancaster University for their hospitality. This research was supported by the NASA Targeted Research and Technology Program, by the NSF National Space Weather Program, and by the Los Alamos National Laboratory LDRD Program.

\section{References}

Bame, S.J., McComas, D.J., Thomsen, M.F., Barraclough, B.L., Elphic, R.C., Glore, J.P., Gosling, J.T., Chavez, J.C., Evans, E.P., Wymer, F.J., 1993. Magnetospheric plasma analyzer for spacecraft with constrained resources. Review of Scientific Instruments 64, 1026.

Borovsky, J.E., Denton, M.H., 2006. The differences between CME-driven storms and CIR-driven storms. Journal of Geophysical Research 111, A07S08.

Borovsky, J.E., Denton, M.H., 2008. A Statistical Look at Plasmaspheric Drainage Plumes. Journal of Geophysical Research, in press, doi:10.1029/2007JA012994.

Borovsky, J.E., Steinberg, J.T., 2006. The "calm before the storm" in CIR/ magnetosphere interactions: occurrence statistics, solar-wind statistics, and magnetospheric preconditioning. Journal of Geophysical Research 111, A07S10.

Borovsky, J.E., Thomsen, M.F., McComas, D.J., 1997. The superdense plasma sheet: plasmaspheric origin, solar-wind origin, or ionospheric origin? Journal of Geophysical Research 102, 22089.

Borovsky, J.E., Thomsen, M.F., Elphic, R.C., 1998a. The driving of the plasma sheet by the solar wind. Journal of Geophysical Research 103, 17617.

Borovsky, J.E., Thomsen, M.F., McComas, D.J., Cayton, T.E., Knipp, D.J., 1998b. Magnetospheric dynamics and mass flow during the November 1993 storm. Journal of Geophysical Research 103, 26373.

Borovsky, J.E., Thomsen, M.F., Elphic, R.C., Cayton, T.E., McComas, D.J., 1998c. The transport of plasma sheet material from the distant tail to geosynchronous orbit. Journal of Geophysical Research 103, 20297.

Denton, M.H., Borovsky, J.E., 2008. Superposed epoch analysis of highspeed-stream effects at geosynchronous orbit: hot plasma, cold plasma, and the solar wind. Journal of Geophysical Research, in press, doi:10.1029/2007JA012998.

Denton, M.H., Borovsky, J.E., Skoug, R.M., Thomsen, M.F., Lavraud, B., Henderson, M.G., McPherron, R.L., Zhang, J.C., Liemohn, M.W., 2006. Geomagnetic storms driven by ICME- and CIR-dominated solar wind. Journal of Geophysical Research 111, A07S07.

Ebihara, Y., Ejiri, M., 2000. Simulation study on fundamental properties of the storm-time ring current. Journal of Geophysical Research 105, 15843

Fujimoto, M., Mukai, T., Matsuoka, A., Saito, Y., Hayakawa, H., Kokubun, S., Lepping, R.P., 2000. Multi-point observations of cold-dense plasma sheet and its relation with tail-LLBL. Advances in Space Research 25, 1607.

Fujimoto, M., Mukai, T., Kokubun, S., 2002. Cold-dense plasma sheet and hot-dense ions in the inner-magnetosphere. Advances in Space Research 30, 2279.

Fujimoto, M., Mukai, T., Kokubun, S., 2005. The structure of the plasma sheet under northward IMF. In: Hoshino, M., Omura, Y., Lanzerotti, L.J. (Eds.), Frontiers in Magnetospheric Plasma Physics. Elsevier, Amsterdam, p. 19

Gussenhoven, M.S., Madden, D., 1990. Monitoring the polar rain over a solar cycle: a polar rain index. Journal of Geophysical Research 95, 10399.

Horne, R.B., Thorne, R.M., 1998. Potential waves for relativistic electron scattering and stochastic acceleration during magnetic storms. Geophysical Research Letters 25, 3011.

Kavanagh, A., Denton, M.H., 2007. High speed solar wind streams and geospace interactions. Astronomy and Geophysics 48, 6.24-6.26.

King, J.H., Papitashvili, N.E., 2005. Solar wind spatial scales in and comparisons of hourly wind and ACE plasma and magnetic field data. Journal of Geophysical Research 110, 2104

Kozyra, J.U., Liemohn, M.W., 2003. Ring current energy input and decay. Space Science Reviews 109, 105.

Lavraud, B., Denton, M.H., Thomsen, M.F., Borovsky, J.E., Friedel, R.H.W., 2005. Superposed epoch analysis of dense plasma access to geosynchronous orbit. Annales de Geophysique 23, 2519.

Lavraud, B., Thomsen, M.F., Borovsky, J.E., Denton, M.H., Pulkkinen, T.I., 2006. Magnetosphere preconditioning under northward IMF: evidence from the study of coronal mass ejection and corotating 
interaction region geoeffectiveness. Journal of Geophysical Research 111, A09208.

Liemohn, M.W., Kozyra, J.U., Thomsen, M.F., Roeder, J.L., Lu, G., Borovsky, J.E., Cayton, T.E., 2001. Dominant role of the asymmetric ring current in producing stormtime Dst*. Journal of Geophysical Research 106, 10883.

Longden, N., Denton, M.H., Honary, F., 2008. Particle precipitation during ICME-driven and CIR-driven geomagnetic storms. Journal of Geophysical Research 113, A06205, doi:10.1029/2007JA012752.

McPherron, R.L., Weygand, J., 2006. The solar wind and geomagnetic activity as a function of time relative to corotating interaction regions. In: Tsurutani, B., McPherron, R., Gonzalez, W., Lu, G., Sobral, J.H.A., Gopalswamy, N. (Eds.), Recurrent Magnetic Storms. American Geophysical Union, Washington, DC, p. 125.

Meredith, N.P., Thorne, R.M., Horne, R.B., Summers, D., Fraser, B.J. Anderson, R.R., 2003. Statistical analysis of relativistic electron energies for cyclotron resonance with EMIC waves observed on CRRES. Journal of Geophysical Research 108, 1250.

Nishino, M.N., Terasawa, T., Hoshino, M., 2002. Increase of the tail plasma content during the northward interplanetary magnetic field intervals: case studies. Journal of Geophysical Research 107, 1261.

Phan, T.D., Lin, R.P., Fuselier, S.A., Fujimoto, M., 2000. Wind observations of mixed magnetosheath-plasma sheet ions deep inside the magnetosphere. Journal of Geophysical Research 105, 5497.

Richardson, I.G., Wibberenz, G., Cane, H.V., 1996. The relationship between recurring cosmic ray depressions and corotating solar wind streams at 1 AU: IMP 8 and Helios 1 and 2 anticoincidence guard rate observations. Journal of Geophysical Research 101, 13483.
Summers, D., Thorne, R.M., 2003. Relativistic electron pitch-angle scattering by electromagnetic ion cyclotron waves during geomagnetic storms. Journal of Geophysical Research 108, 1143.

Terasawa, T., Fujimoto, M., Mukai, t., Shinohara, I., Saito, Y., Yamamoto, T. Machida, S., Kokubun, S., Lazarus, A.J., Steinberg, J.T., Lepping, R.P., 1997. Solar wind control of density and temperature in the nearEarth plasma sheet: WIND/GEOTAIL collaboration. Geophysical Research Letters 24, 935.

Thomsen, M.F., 2004. Why Kp is such a good measure of magnetospheric convection. Space Weather 2, S11004.

Thomsen, M.F., Borovsky, J.E., McComas, D.J., Elphic, R.C., Maurice, S. 1998a. Magnetospheric response to the CME passage of January $10-11,1997$, as seen at geosynchronous orbit. Geophysical Research Letters 25, 2545

Thomsen, M.F., McComas, D.J., Borovsky, J.E., Elphic, R.C., 1998b. The magnetospheric trough. In: Horwitz, J.L., Gallagher, D.L., Peterson, W.K. (Eds.), Geospace Mass Transport and Energy Flow: Results fro the International Solar-Terrestrial Physics Program. American Geophysical Union, Washington, DC, p. 355.

Thomsen, M.F., Borovsky, J.E., Skoug, R.M., Smith, C.W., 2003. Delivery of cold, dense plasma sheet material into the near-Earth region. Journal of Geophysical Research 108, 1151.

Tsurutani, B.T., et al., 2006. Corotating solar wind streams and recurrent geomagnetic activity: a review. Journal of Geophysical Research. 111 A07S01, doi:10.1029/2005JA011273.

Wilcox, J.M., Ness, N.F., 1967. Solar source of the interplanetary sector structure. Solar Physics 1, 437 